\title{
Geometric Derivative Filter for Image Enhancement
}

\author{
Hamid Hassanpour
}

Email: h.hassanpour67@gmail.com

\begin{abstract}
In this paper, we introduce concept of geometric derivative filter as a new method for enhancing objects in images. It utilizes Euclidean distance filter for magnifying appearance of objects and structures in images. This filter can be used solely for enhancing quality of image or can be used as a pre-processing step for deep learning applications. This filter is applicable in places where image is hazy or transition between objects in image is smooth, i.e., when objects in image are not clear.
\end{abstract}

Keywords: image processing, image filters, Euclidean distance filter

\section{Introduction}

Image filters usually have computation on time or frequency domains. Geometrical aspect of images is the missing part of image processing. In this article, in order to enhance quality of an image, we use derivative terms, which means difference between pixel values in two locations of an image. We magnify this difference using Euclidean distance filter described in Hassanpour, H. (2021). In this way, by magnification of this difference, i.e., by applying Euclidian distance filter as weights to these differences, pixel value at the centre of location where the filter applied is calculated. The filter is swept through all pixels in image and then final images are depicted in results section.

\section{Methodology}

Geometric derivative filter is a rectangular filter were for calculating its values two steps are implemented. In the first step, Euclidean distance filter values are calculated. In the second step derivative term is calculated. At the end, derivative term is weighted with Euclidean distance filter values and the pixel resides at the centre of location where the filter is applied will be updated. In order to calculate derivate term, at the location of the image where the filter is applying, difference between pixel value in centre of that location and pixels around it which are resides inside $n \times n$ filter box is calculated. Mathematical illustration of the concept is as follows:

$$
\begin{gathered}
D_{x}=x-X \\
F=G * D_{x} \\
y=x+\sum_{n \times n} F
\end{gathered}
$$

In this formulation, $x$ is pixel value at the centre of location where the filter is applied. $X$ is the image box in the location that the filter is applying. It is equivalent to a $n \times n$ matrix. In order to have $n \times n$ matrix of derivative term, that is named as $D_{x}, x$ need to be 
broadcasted to a $n \times n$ matrix. $G$ is Euclidian distance filter with the same shape as our proposed geometrical derivative filter, which is donated with $F$. The product operation $\left({ }^{*}\right)$ is element-wise. In order to have value of a pixel after applying the filter, geometric derivative filter values, F, need to be summed up across both rows and columns. The output of the filter which is pixel value at the centre of location where the filter is applying, which is named $y$, is the summation of $\sum_{n \times n} F$ term and pixel's original value $x$.

\section{Results}

Geometrical derivative filter is applicable in hazy images, where transition between objects is not clear or this transition is very smooth. In these situations, object detection, both for eyes and computer applications like deep learning framework is very hard. One of important applications of this filter is for enhancing X-ray images, both for technical experts and for computer applications in this area.

Figure one illustrates two examples of application of this filter. As both pictures show, applying the filter enhances bones, makes them become more separable and observable. Source code in available in Geometric derivative filter implementation (2021).

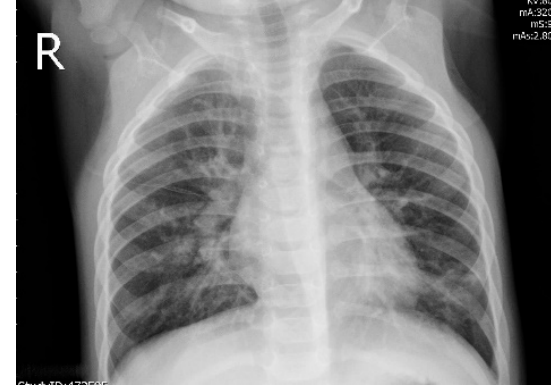

$a$

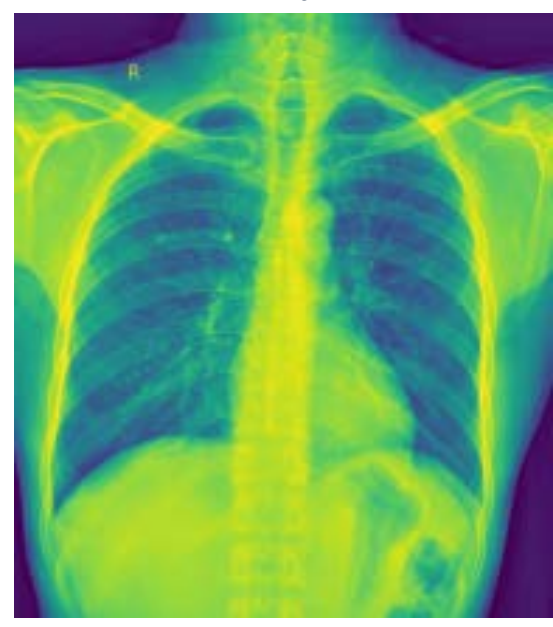

C

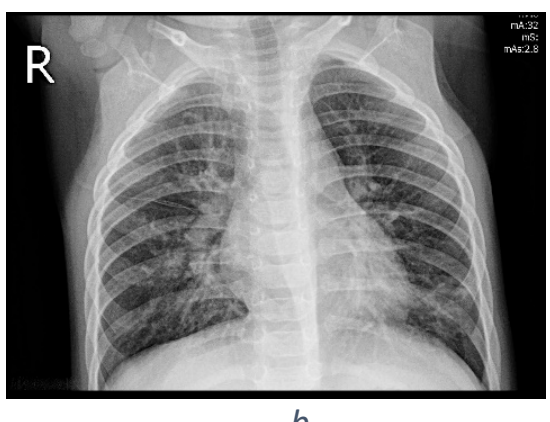

$b$

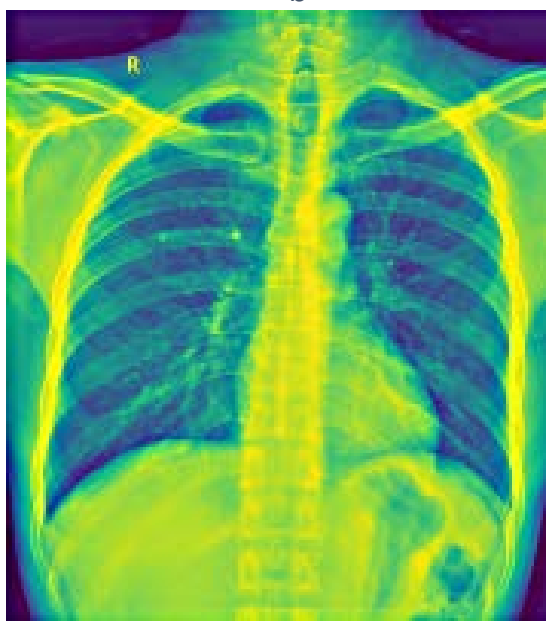

d

Figure 1: Comparison of original images (a) and (c) with 10*10 filtered images (b) and (d) 
Figure 2 shows another example with regular hazy image. The result indicated in picture b, where objects in the image become more apparent and separable from its surroundings.

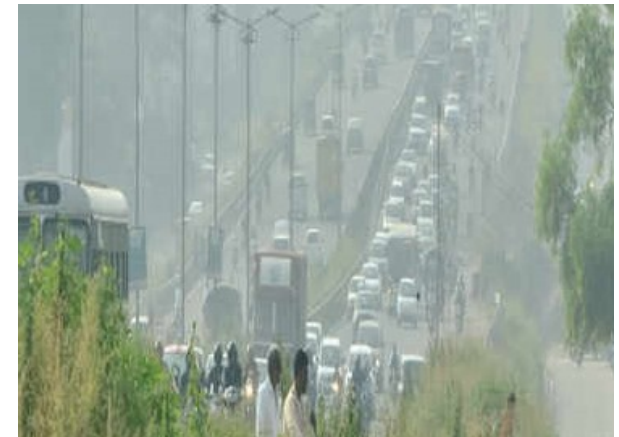

a

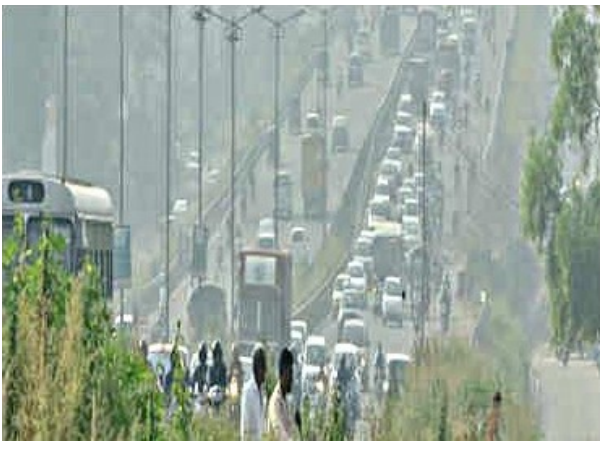

$b$

Figure 2: Regular hazy image (b) and after applying 10*10 filter(b)

\section{Reference}

Geometric derivative filter implementation (2021) [online] https://github.com/HamidHassanpour/Geometric-Derivative-Filter (Accessed 6 February 2021)

Hassanpour, H. (2021) Euclidian Distance Filter for Image Processing https://www.techrxiv.org/articles/preprint/Euclidean_Distance_Filter_for_Image_Pro cessing/13664615 (Accessed 6 February 2021) 